\title{
Cell Survival Is Regulated via SOX9/BCL2L1 Axis in HCT-116 Colorectal Cancer Cell Line
}

\author{
Erik Lizárraga-Verdugo, ${ }^{1}$ Erika Ruiz-García $\mathbb{D}^{2},{ }^{2}$ César López-Camarillo $\mathbb{D}^{3},{ }^{3}$ \\ Mercedes Bermúdez, ${ }^{1}$ Mariana Avendaño-Félix, ${ }^{1}$ Rosalío Ramos-Payán $\left(\mathbb{D},{ }^{1}\right.$ \\ Geovanni Romero-Quintana, ${ }^{1}$ Alfredo Ayala-Ham, ${ }^{4}$ Carlos Villegas-Mercado, ${ }^{4}$ \\ Carlos Pérez-Plasencia $\mathbb{D}^{\mathbb{D}},{ }^{2}$ and Maribel Aguilar-Medina $\mathbb{D}^{1}$ \\ ${ }^{1}$ Facultad de Ciencias Químico Biológicas, Universidad Autónoma de Sinaloa, Culiacán, Sinaloa, Mexico \\ ${ }^{2}$ Instituto Nacional de Cancerología, CDMX, Mexico \\ ${ }^{3}$ Posgrado en Ciencias Genómicas, Universidad Autónoma de La Ciudad de México, CDMX, Mexico \\ ${ }^{4}$ Facultad de Odontología, Universidad Autónoma de Sinaloa, Culiacán, Sinaloa, Mexico \\ Correspondence should be addressed to Maribel Aguilar-Medina; maribelaguilar@uas.edu.mx
}

Received 18 January 2020; Revised 20 March 2020; Accepted 10 April 2020; Published 29 April 2020

Academic Editor: Reza Izadpanah

Copyright (c) 2020 Erik Lizárraga-Verdugo et al. This is an open access article distributed under the Creative Commons Attribution License, which permits unrestricted use, distribution, and reproduction in any medium, provided the original work is properly cited.

Colorectal cancer (CRC) is one of the most frequent types of malignancies and one of the major causes of cancer-related death worldwide. Sex-determining region Y (SRY)-box 9 protein (SOX9) is a member of the SOX family of transcription factors which are involved in the regulation of differentiation and development. Recently, several reports suggest an important role of SOX9 in tumorigenesis since its overexpression correlates with tumor progression and poor outcome in several types of cancer; however, its role in CRC is not clear until now. Therefore, in this work, we searched for novel SOX9-regulated genes involved in cell survival of CRC. We silenced SOX9 in the poorly differentiated HCT-116 cell line, using a specific siRNA, to identify differential expressed genes by DNA microarrays and analyzed the role or candidate genes in apoptosis and autophagy. Transcriptome analysis showed that diverse cellular pathways, associated with CRC carcinogenesis such as Wnt/ $\beta$-catenin, MAPK, TGF- $\beta$, and mTOR, were modulated after SOX9 silencing. Interestingly, we found that SOX9 silencing promotes downregulation of BCL2L1 and overexpression of CASP3, proteins related to apoptosis, which was further confirmed in SW-480, a moderated-differentiated cell line, but not in HT-29, well-differentiated cell line. Moreover, inhibition of BCL2L1 by ABT-737 (BH3 mimetic) in SOX9-silenced HCT-116 cells resulted in an increased apoptosis percentage. However, downregulation of BCL2L1 was not enough to induce autophagy. This is the first report, suggesting that cell survival in poorly and moderated-differentiated CRC cells lines is regulated by SOX9/BCL2L1 axis, but not in well-differentiated cell lines.

\section{Introduction}

Colorectal cancer (CRC) is a multifactorial disease that results from lifestyle, genetic, and environmental factors [1], and it is considered as one of the major causes of cancerrelated death worldwide [2]. Intestinal epithelium exhibits a high turnover rate, which favors carcinogenesis [3]; in contrast, stem cells from intestinal crypts are responsible of tissue maintaining and homeostasis since they show a low rate of cell death, a process regulated by programmed cell death [4] through different mechanisms such as apoptosis, autophagy, and necroptosis [5]. Programmed cell death resistance is considered as a hallmark of cancer [6].

There are two basic signaling pathways that regulate apoptosis: extrinsic and intrinsic [5, 7]. The extrinsic pathway is stimulated by interaction of ligands to cell surface-exposed death receptors that culminates in caspase8 activation, for example, the tumor necrosis factor receptors (TNFRs) and their respective protein TNF family ligand, among others [8]. On the contrary, the intrinsic pathway is mitochondria-dependent mediated by intracellular signals in response to different stress conditions including 
chemotherapeutic agents, DNA damage, growth factor deprivation, and oxidative stress, among others [9]. The activation of intrinsic apoptosis pathway drives to apoptosome formation, which is composed of procaspase-9, apoptotic protease-activating factor (Apaf-1), and cytochrome c, that concludes in caspase-9 activation [5]. This process is led by Bcl-2 family members' proteins that can be divided into two groups: proapoptotic or antisurvival (Bax, Bak, Bid, Bad, and Bok) and antiapoptotic or prosurvival (Bcl-2, Bcl-w, and $\mathrm{Bcl}-\mathrm{xL}$ ) [10]. Bax drives to apoptosome formation and caspase-3 activation, promoting apoptosis and has been reported that Bcl-xL, encoded by BCL2L1 (BCL2-like 1) gene [11], is able to stabilize the mitochondrial localization of Bax, maintaining it in an inactive state [12], which is a mechanism implicated in cell survival in different tumor types [13].

Several signaling pathways have key roles in the regulation of cell survival within tumor tissues, for instance, $\mathrm{Wnt} / \beta$-catenin signaling pathway, which in turn is regulated by SOX9 through two main mechanisms: $\beta \beta$-catenin degradation and transcriptional activity inhibition [14-16]. SOX9 is a member of SOX (SRY (sex-determining region Y)related high mobility group (HMG) box) family and works as a transcription factor that plays a central role in the development and differentiation of multiple cell lineage [17]. Overexpression and mutation of SOX9 increases cell proliferation, invasiveness, and metastasis in several types of cancer [18-21]; nonetheless, little is known about SOX9 and its importance in cell survival in CRC. Therefore, the aim of this study was to identify novel genes regulated by SOX9 and their role in cell survival in CRC.

\section{Materials and Methods}

2.1. Cell Culture. The human colorectal cancer cell lines HCT-116, SW-480, Caco-2, HT-29, and nontumorigenic cell line CCD-18Co were obtained from American Type Culture Collection (ATCC). HCT-116 and HT29 were cultured in DMEM/F12 (Gibco) supplemented with $10 \%$ fetal bovine serum (FBS) and 1\% penicillin/streptomycin. SW-480 was cultured in RPMI 1640 (Gibco) supplemented with 15\% FBS and $1 \%$ penicillin/streptomycin. CCD-18Co and Caco-2 were grown in DMEM/F12 supplemented with 10\% FBS, 1\% penicillin/streptomycin, and $1 \%$ nonessential amino acids (NEAA). All cell lines were incubated at $37^{\circ} \mathrm{C}$ with $5 \% \mathrm{CO}_{2}$ in a humidified atmosphere.

2.2. SOX9 Silencing. HCT-116 cell line was seeded at a density of $3 \times 10^{5}$ cells per well into a 6 -well plate, and after $70 \%$ of confluence was reached, cells were transfected with $30 \mathrm{nM}$ of Silencer Select siRNA (ID: s532658, Thermo Fisher Scientific) specific to SOX9, using lipofectamine RNAiMAX reagent (Invitrogen) according to the manufacturer's protocol. SOX9 silencing confirmation was assessed by RTqPCR at $24 \mathrm{~h}$ posttransfection.

2.3. RNA Isolation and RT-qPCR. Total RNA was isolated by TRIzol Reagent (Invitrogen) method following the manufacturer's instructions. cDNA synthesis was performed with $2 \mu \mathrm{g}$ of total RNA using High-Capacity cDNA Reverse Transcription Kit (Thermo Fisher Scientific). Real-time PCR was performed using TaqMan Gene Expression Assays (Applied Biosystems) and TaqMan ${ }^{\mathrm{TM}}$ Universal PCR Master Mix (Thermo Fisher Scientific) for SOX9 (Hs00165814_m1), BCL2L1 (Hs00236329_m1), and CASP3 (Hs00234387_m1). The mixtures were incubated at $50^{\circ} \mathrm{C}$ for $2 \mathrm{~min}, 95^{\circ} \mathrm{C} 10 \mathrm{~min}$, followed by 40 cycles of $95^{\circ} \mathrm{C}$ for $15 \mathrm{~s}$ and $60^{\circ} \mathrm{C}$ for $1 \mathrm{~min}$. Gene expression was measured in triplicate, and data were analyzed using $2^{-\Delta \Delta C T}$ method. ACTB (Hs01060665_g1) was used as endogenous control.

2.4. Microarray Hybridization, Detection, and Preprocessing. Whole-genome transcriptome analysis was performed using Clariom D arrays (Affymetrix GeneChip) following manufacturer's instructions. In brief, the Affymetrix GeneChip WT Pico Kit was used for cDNA preparation and biotin labeling. cRNA was purified using an Affymetrix magnetic bead protocol. The Affymetrix GeneChip ${ }^{\mathrm{TM}}$ Hybridization, Wash, and Stain Kit was used for array processing. Arrays were incubated for $16 \mathrm{~h}$ in an Affymetrix GeneChip 645 hybridization oven at $45^{\circ} \mathrm{C}$ with rotation at $60 \mathrm{rpm}$. Fluorescence was amplified by adding biotinylated antistreptavidin and an additional aliquot of streptavidinphycoerythrin stain. A confocal scanner (Affymetrix GeneChip Scanner $30007 \mathrm{G}$ plus) was used to collect the fluorescence signal at $3 \mu \mathrm{m}$ resolution after excitation at $570 \mathrm{~nm}$. The average signal from two sequential scans was calculated for each microarray.

2.5. Microarray Data Analysis. Microarray data analysis was performed using Partek Genomic Suite v8.0. All samples were normalized with Robust Multiarray Average (RMA) [22], which includes background correction, normalization, and calculation of expression values. Differential expression analysis was performed using ANOVA one way. We selected differentially expressed (DE) genes between groups based on a fold-change of 2 in absolute value, and Benjamini and Hochberg false discovery rate [23] was applied for multiple hypotheses testing, and the genes with an adjusted $p<0.01$ were accepted. The enrichment analysis with DAVID (Database for Annotation, Visualization, and Integrated Discovery) [24, 25], and Partek Genomic Suite v8.0 was performed on each list of selected genes. Partek Genomic Suite was also used for pathway analysis.

2.6. In Silico Analysis. Dataset from The Cancer Genome Atlas (TCGA) was queried and analyzed using the Gene Expression Profiling and Interactive Analyses (GEPIA) [26] platform (http://gepia.cancer-pku.cn/). A total of 275 CRC tissues were included and compared with 349 normal adjacent tissues in order to compare SOX9 expression. Finally, Partek Genomic Suite was also used for pathway analysis, and interactome analysis was developed in String (https:// string-db.org/cgi/network.pl?taskId=Y8RKNUbzndpT) in order to identify association between DE genes. 
2.7. Flow Cytometry Apoptosis Analysis. A total of 90,000 cells were seeded in a 24 -wells plate and incubated at $37^{\circ} \mathrm{C}$ for $24 \mathrm{~h}$, and then the fresh medium containing $5 \mu \mathrm{M}$ ABT737 (Abcam, ab141336), a BH3 mimetic, was added for $48 \mathrm{~h}$ to inhibit BCL2L1 function. According to the recommended protocol, the apoptosis rates were analyzed by Annexin-V$\mathrm{PE}$ and 7-AAD double-staining method based on the PE Annexin V Apoptosis Detection Kit I (BD Pharmingen, 559763) through flow cytometry using a BD Accuri C6 (BD Biosciences, San Jose, CA, USA). Data were analyzed using FlowJo v.10.

2.8. Immunofluorescence Analysis. 80,000 cells were grown in round-glass cover slips into a 24 -well plate. Immunofluorescence was performed after $48 \mathrm{~h}$ posttransfection. In brief, cells were washed with cold 1X PBS and fixated with $4 \% p$-formaldehyde for $10 \mathrm{~min}$. Samples were washed three times with $1 \mathrm{X}$ PBS and permeabilized with $0.1 \%$ Triton X-100 for $10 \mathrm{~min}$. After permeabilization, samples were washed and blocked with $22.52 \mathrm{mg} / \mathrm{mL}$ glycine and $1 \%$ bovine serum albumin. Samples were hybridized with $1: 200$ rabbit anti-human SOX9 (Abcam ab185966) or 1:500 rabbit anti-human LC3B (Abcam ab51520) for $1 \mathrm{~h}$ at room temperature and then washed and incubated with $1: 1,000$ secondary antibody donkey anti-rabbit IgG Alexa 647 (Abcam ab150075) for $1 \mathrm{~h}$ at room temperature. Anti-PGA3 antibody (7G3) (Abcam ab50123) and anti-GAPDH antibody (Abcam ab9485) were used as an isotype control and loading control, respectively. Finally, samples were washed and mounted with Fluoroshield with DAPI (Sigma). Images were obtained at a 40x magnification in a Leica LSCM (TCS SP8 Leica Microsystems). Mean of fluorescent intensity was calculated by choosing five ROIs in each experiment using Leica Application Suite $X$.

2.9. Statistical Analysis. Experiments were performed three times by triplicate, and results were represented as mean \pm S.D. The student's $t$-test was used when comparing two parametric variables; ANOVA and multiple comparisons Tukey's test were used for 3 or more parametric variables. $p<0.05$ was considered as statistically significant.

\section{Results and Discussion}

3.1. SOX9 Is Overexpressed in CRC Tumors and Cell Lines. In silico analysis of $275 \mathrm{CRC}$ tumor tissues, of patients with colon adenocarcinoma from TCGA database, showed a higher SOX9 expression levels ( $\operatorname{LogFch} 3.0$ ) in comparison with 349 healthy adjacent tissues $(p<0.01)$ (Figure $1(a)$ ). These results show the same pattern when compared with other types of cancer such as renal cell carcinoma (RCC). Besides, overexpression of SOX9 is related to clinicopathological characteristics, such as the advanced pathological grade and clinical stage. Also, SOX9 is an independent predictor factor for the survival of RCC patients in the TCGA dataset [27].

In our study, we found that SOX9 is overexpressed in HCT-116 $(p=0.0061), \quad$ HT-29 $\quad(p=0.0151), \quad$ Caco-2 $(p=0.0269)$, and SW-480 $(p=0.0405)$ CRC cell lines (Figure 1(b)). In particular, the poorly differentiated HCT116 showed the highest expression levels $(\operatorname{LogFch}=173.56)$; thus, it was selected for downstream analysis. Interestingly, SOX9 expression has been related with lower overall survival, tumor size, tumor progression, metastasis, and cancer cell plasticity in CRC samples at advanced stages [28-30].

3.2. SOX9 Silencing Induces Important Changes in Transcriptome Expression of HCT-116 Cells. SOX9 protein level was decreased $84.4 \%(p=0.001)$ in transfected HCT-116 $\left(\right.$ HCT-116 ${ }^{\text {sisOX9 }}$ ) (Figures $1(\mathrm{c})$ and 1(d)). Comparable results were obtained at mRNA expression level $(p<0.002)$ (Figure 1(e)).

To gain insight into the biological functions of SOX9, the gene expression profile of HCT- $116^{\text {sisOX9 }}$ cells was obtained. Analysis of the original normalized microarrays dataset revealed a total of 369 overexpressed and 151 downregulated genes (LogFch $>2$ or $<-2$, adjusted $p<0.01$ ) (Figures 2 (a) and $2(b)$ ). The full list of deregulated genes is provided in Supplementary Materials (Table S1). Functional analysis reported seven clusters with an enrichment score (ES) greater than 2: nucleosome core (ES 8.71), transcription regulation (ES 7.63), apoptosis regulation (ES 3.23), betacatenin-TFC complex assembly (ES 2.97), cell cycle (ES 2.8), zing finger (ES 2.46), and DNA repair (ES 2.37) (data not shown). The highest changes in gene expression were in APC (LogFch 19.9) and MYC (LogFch-3.0). Interestingly, 25 histones were downregulated, while transcriptional regulators such as DBF4, ATF2, ATRX, and AFF4 were overexpressed. As expected, pathways with overrepresentation were CRC (Figure S1) and WNT signaling pathways (Figure S2), in which APC was present. This is relevant since it is well known that loss of APC function activates the cascade of events that ultimately lead to malignant transformation [31].

Interestingly, string interactome analysis of Table 1 genes (Figure S3) grouped in apoptosis cluster showed a network associating SOX9 with BCL2L1 through JUN. SOX9 is linked directly to CASP according to text mining, and association of BCL2L1 and CASP3 is established based on experimental results. Moreover, interactome suggests TP53 as a master molecule since it interacts directly with BCL2L1, CASP3, APC, MYC, and JUN. Previously, BCL2L1 and CASP3 have been described as cell survival mediators [32].

3.3. BCL2L1 and CASP3 Gene Expression Levels Are Regulated by SOX9 in HCT-116 Cells. For microarrays data validation, we evaluated gene expression of apoptosis regulators in independent HCT-116 transfection assays. We found that after silencing of SOX9 $(p<0.0001)$, BCL2L1 was $25 \%$ downregulated $(p=0.037)$. Besides, CASP3 enhanced its expression level by $75 \%(p=0.0005)$ (Figure $2(c))$, corroborating microarrays' findings. Interestingly, there are no reports indicating the regulation of prosurvival BCL2L1 and antisurvival CASP3 by SOX9 in CRC.

There is evidence that Bcl-2, a member of BCL2 family, is capable to regulate SOX9 through MEK-ERK1/2, 


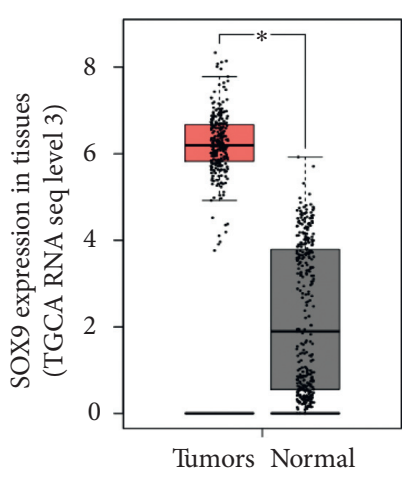

(a)

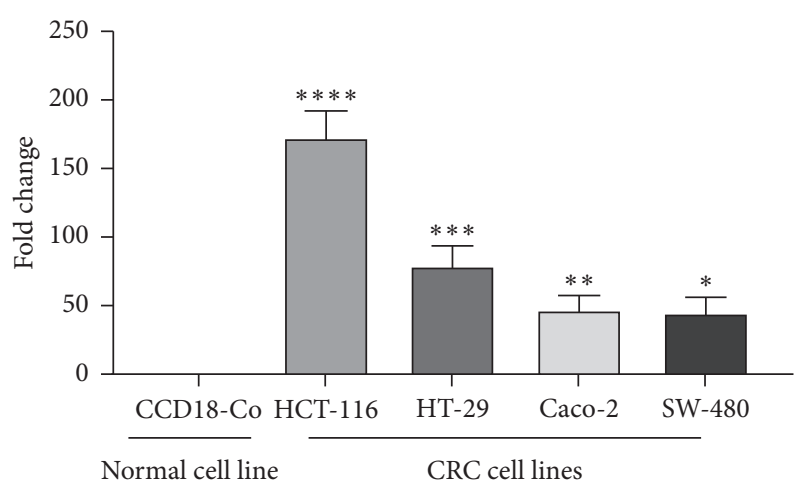

(b)
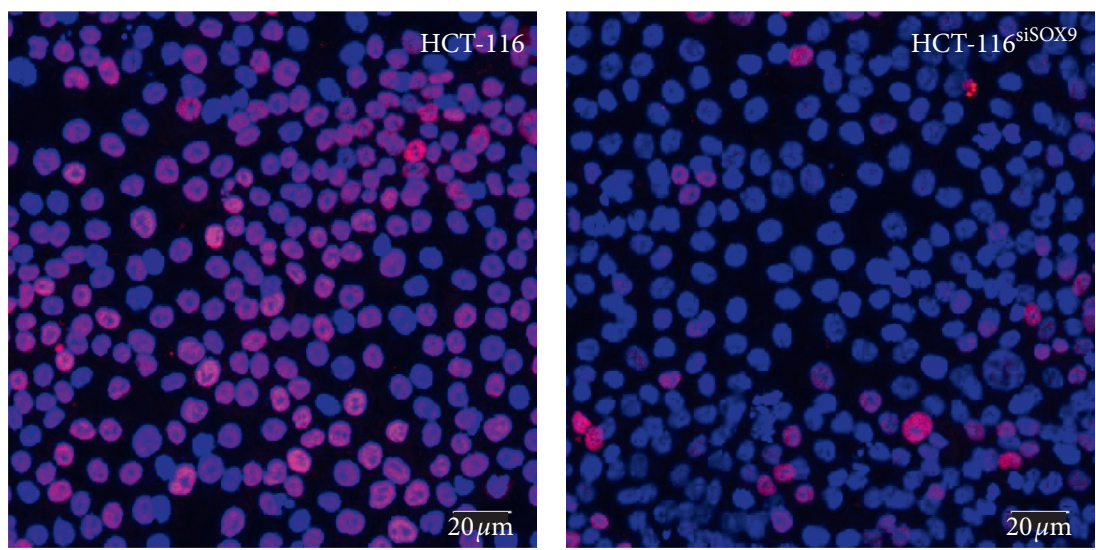

DAPI

SOX9

(c)

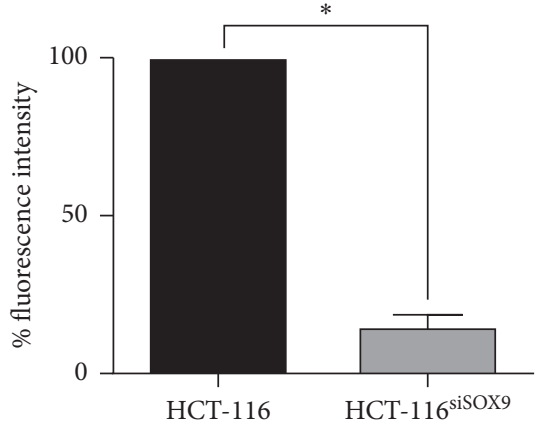

(d)

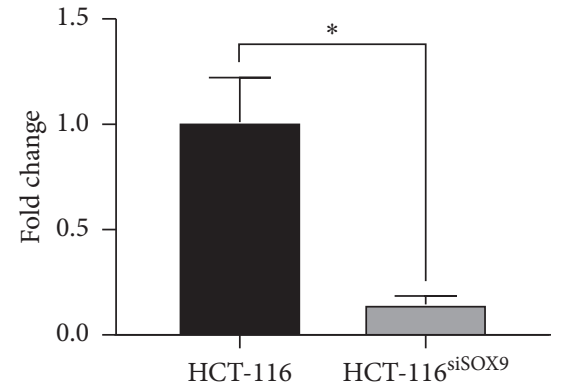

(e)

FIGURE 1: SOX9 is overexpressed in tumors and CRC cells lines. (a) TCGA datasets in silico analysis showed that SOX9 is overexpressed in colon cancer tissues in comparison with adjacent normal samples $\left({ }^{*} p<0.001\right)$. (b) Quantitative RT-qPCR showed that SOX9 is overexpressed in all studied CRC cell lines in comparison with the nontumorigenic CCD-18Co cell line (all $p<0.001$ ). (c) Immunofluorescence assays showed that nuclear SOX9 expression is highly diminished in HCT-116 SOX9-silenced cells. (d) Fluorescence intensity mean in HCT-116 SOX9-silenced cells compared with control $\left({ }^{*} p=0.001\right)$. (e) RT-qPCR analysis confirmed SOX9 silencing in HCT-116 cells $\left({ }^{*} p=0.002\right)$.

maintaining the phenotype of differentiated chondrocytes [33]. Our results suggest that cell survival could be negatively regulated by SOX9 expression since its downregulation promotes a decrease of BCL2L1 and an increase of CASP3 in HCT-116. Accordingly, downregulation of SOX9 produces an increased apoptosis due to Bcl-xL decrease in human chordoma cell lines [34]. SOX9 downregulation in cholangiocarcinoma enhances expression of apoptosis effectors such as CASP3 and CASP8, suggesting that SOX9 might play an important role in the process of apoptosis [35]. Remarkably, SOX9 modification does not affect caspases activities in other types of cancer such as basal cell and prostate carcinoma [36, 37]. Altogether, these data might suggest that SOX9 could regulate induced cell death by 


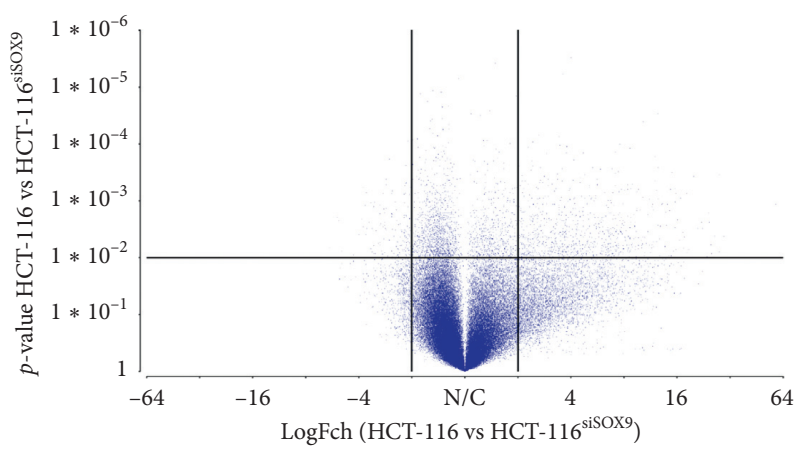

(a)
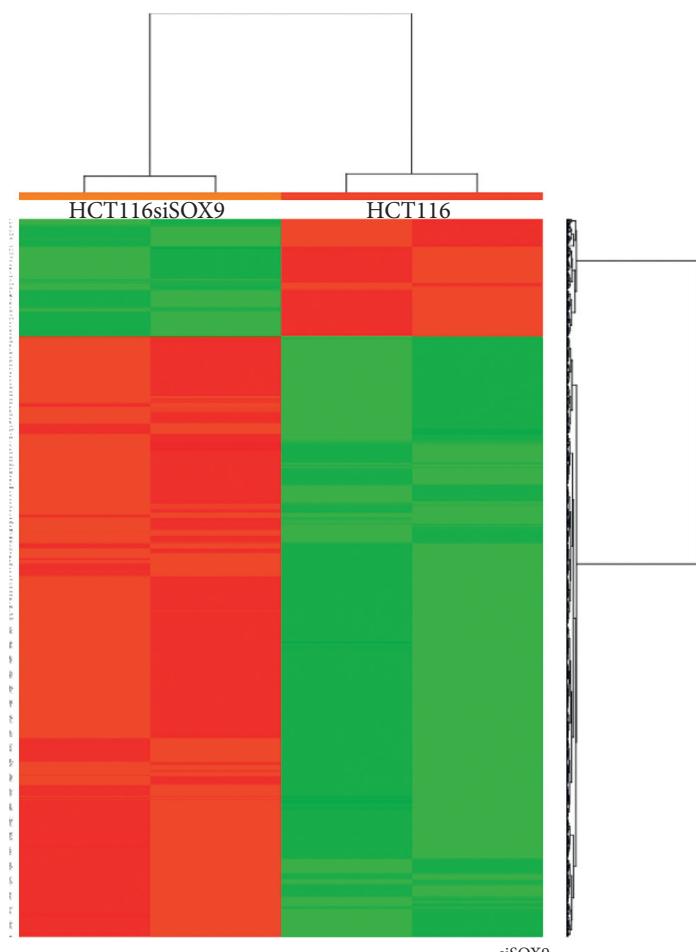

Hierarchical (HCT-116 vs HCT-116 $6^{\text {sisox9 }}$

(b)

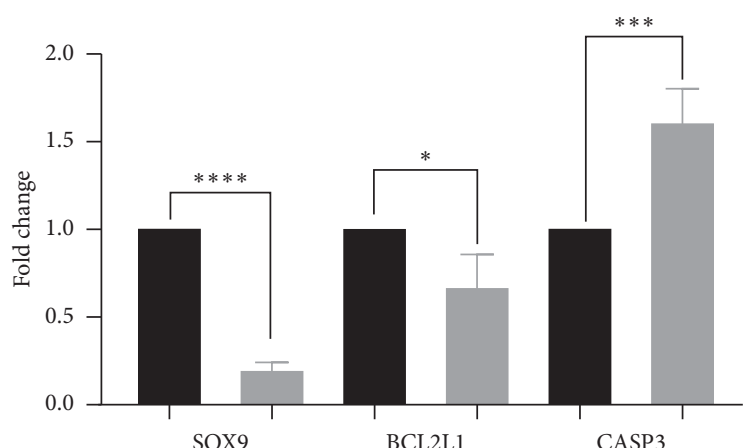

HCT-116

HCT-116 $6^{\text {sisox } 9}$

(c)

FIGURE 2: SOX9 silencing deregulates several signaling pathways. Transcriptome profiles of HCT-116 SOX9-silenced and nonsilenced were compared, based on microarray data. (a) In volcano plot, points represent significantly upregulated and downregulated mRNAs in HCT116siSOX9 with a 2.0-LogFch. (b) Two-dimensional hierarchical clustering of distinguishable mRNAs expression profiles in both groups. Red: higher expression levels; green: lower expression levels. (c) RT-qPCR analysis for microarray data validation confirmed that SOX9 silencing modulates gene expression of selected candidates' genes: SOX9 $\left({ }^{* * * *} p<0.0001\right)$, BCL2L1 $\left({ }^{*} p=0.37\right)$, CASP3 $\left({ }^{* * *} p=0.0005\right)$, and NF1 (** $p<0.0007)$, in an independent experiment. 
TABLE 1: Differentially expressed apoptosis-related genes.

\begin{tabular}{lcc}
\hline Gene & Fold change & $p$ value \\
\hline BLID & 6.86524 & $6.81 E-05$ \\
SOS1 & 6.34372 & 0.00854877 \\
TAOK1 & 6.4378 & 0.00779361 \\
CASP3 & 3.37851 & 0.00672809 \\
CASP8AP2 & 3.64127 & 0.00193459 \\
CHUK & 3.57394 & 0.0058005 \\
HIPK3 & 6.31868 & 0.00480743 \\
APC & 19.9097 & 0.00037474 \\
BCL2L1 & -2.26984 & 0.00601934 \\
CHAC1 & -5.85796 & 0.00664003 \\
E2F1 & -3.63746 & 0.00398582 \\
JUN & -2.25396 & 0.0092463 \\
APEX1 & -2.28963 & 0.00293408 \\
BEX2 & -2.12243 & 0.00385886 \\
TP53 & -3.04873 & 0.00540116 \\
MYC & -3.00718 & 0.00249873 \\
PDPK1 & -2.05947 & 0.00413932 \\
\hline
\end{tabular}

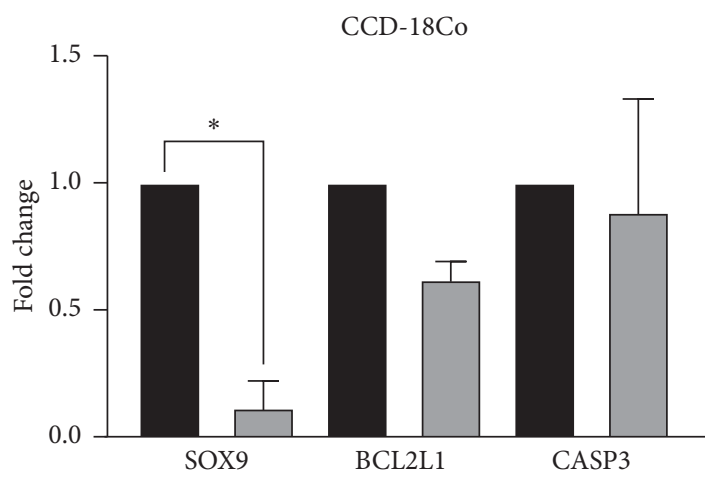

HCT-116

HCT-116 $6^{\text {sisox9 }}$

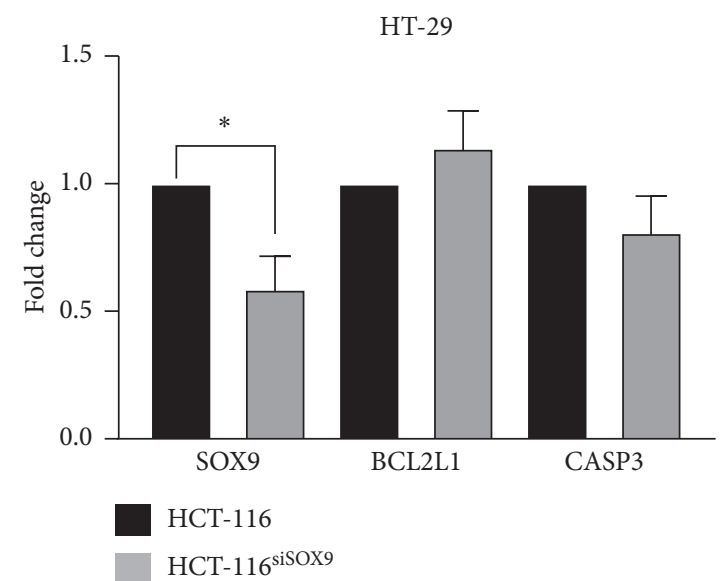

(b)

(a)

SW-480

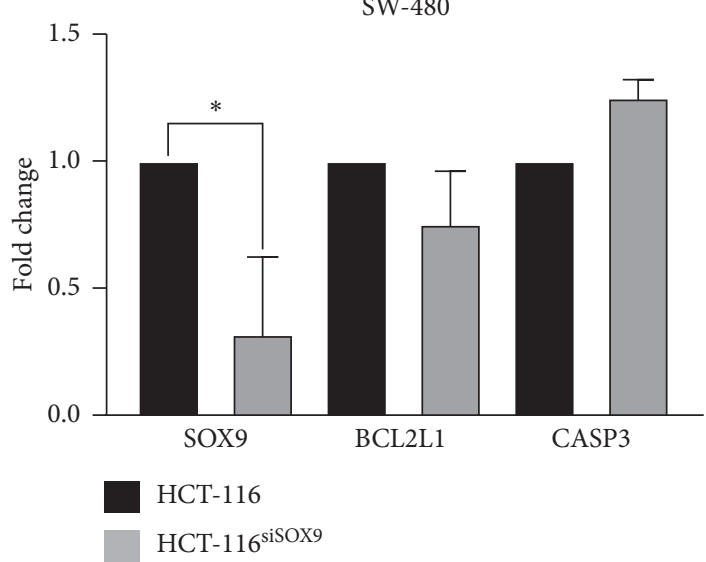

(c)

FIGURE 3: SOX9 regulates BCL2L1 and CASP3 in undifferentiated CRC cell lines. Quantitative RT-qPCR showed that, after SOX9 silencing, BCL2L1 and CASP3 did not change in well-differentiated (a) normal CCD-18Co and (b) HT-29 cell lines, but a tendency similar to HCT116 was seen in the moderate-differentiated SW-480 (c). However, these changes were not statistically significant. For SOX9 silencing in all cell lines, ${ }^{*} p<0.01$. 

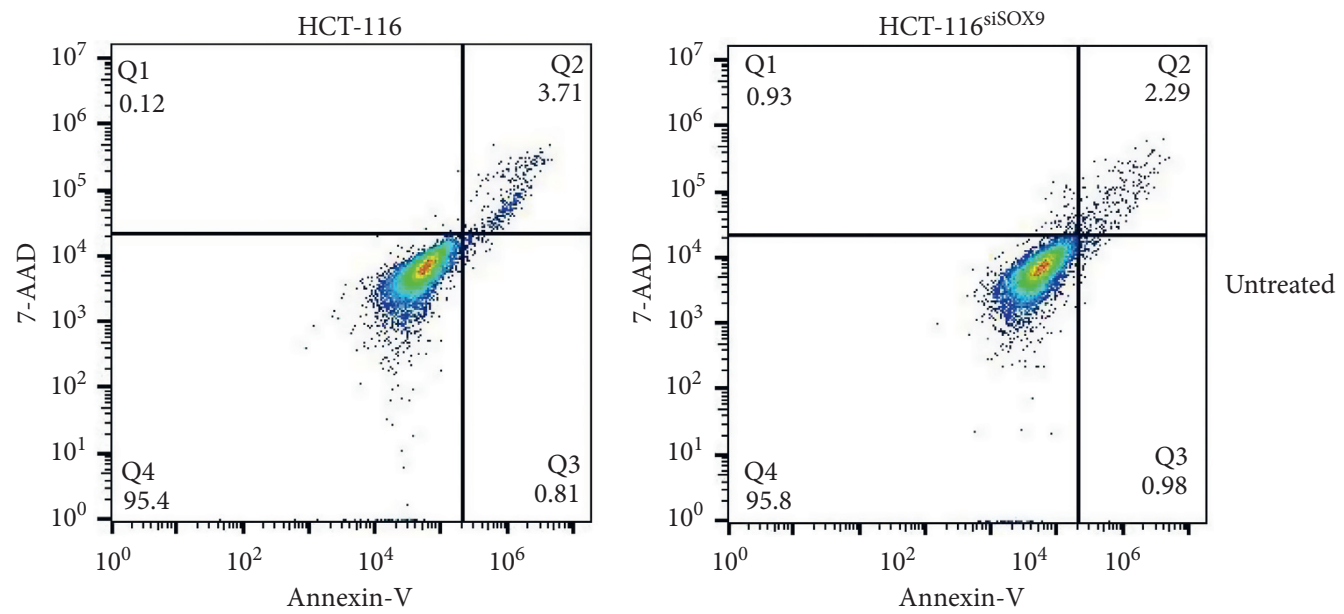

(a)
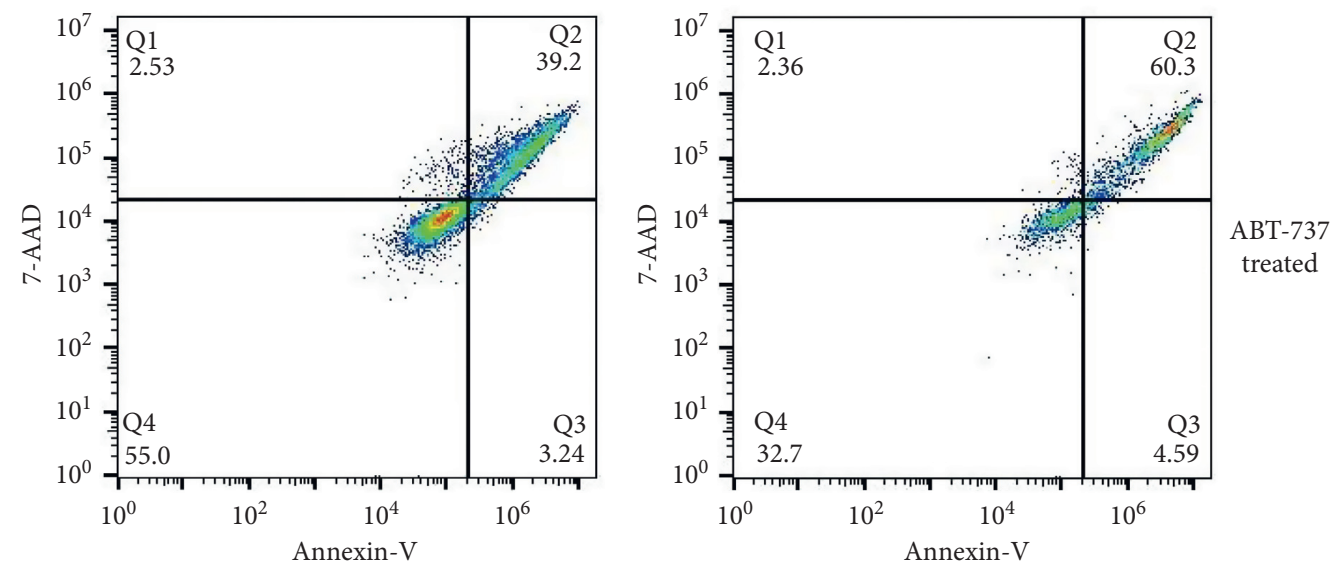

(b)

FIgURE 4: BCL2L1 inhibition increase apoptosis SOX9-silenced HCT-116 cells. Flow cytometry analysis for apoptosis detection through Annexin V and 7-AAD. (a) HCT-116 and HCT-116 ${ }^{\text {sisox } 9}$ untreated. (b) HCT-116 and HCT-116 ${ }^{\text {sisox9 }}$ treated with ABT-737.

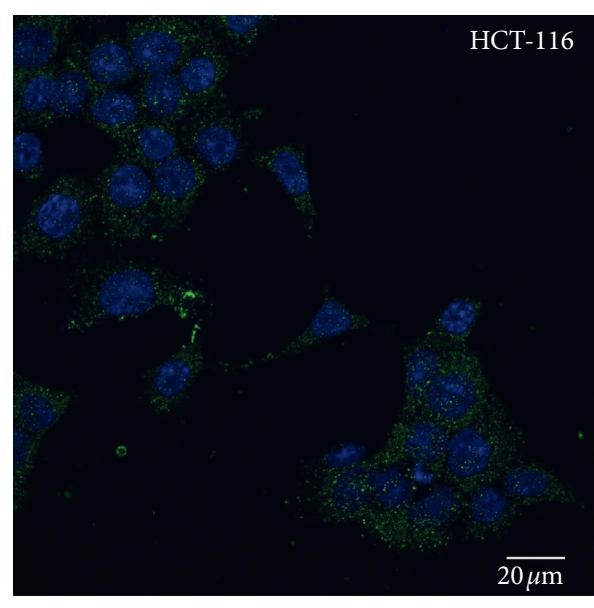

LC3

DAPI

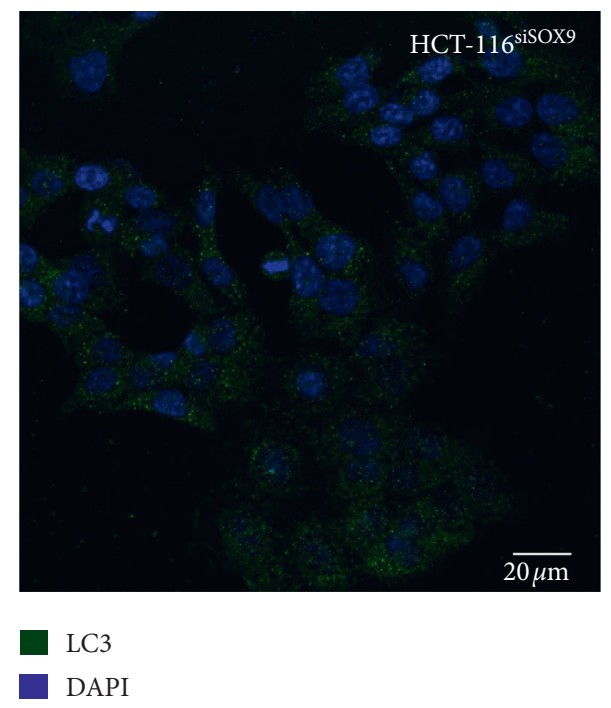

(b)

FIGURE 5: Immunofluorescence assays for autophagy. Autophagosome induction by SOX9/BCL2L1 axis. Micrographies of LC3 protein in HCT-116 and SOX9-silenced HCT-116 cells showed no differences between the groups. 
directly affecting both CASP3 and BCL2L1 expression in HCT-116.

3.4. SOX9/BCL2L1 Axis Is Present in Poorly Diferenciated CRC Cell Lines. SOX9 regulation of BCL2L1 and CASP3 was evaluated in HT-29 (well-differentiated) and SW-480 (moderate-differentiated) CRC cell lines (Figure 3). We found that, after SOX9 silencing, BCL2L1 and CASP3 did not change in differentiated CCD-18Co and HT-29 (Figures 3(a) and 3(b)), but a similar tendency to that seen in HCT-116 was found in SW-480; however, these changes were not statistically significant (Figure 3(c)).

These data are in agreement with the previous report showing an overexpression of BCL2L1 and downregulation of CASP3 in tumorigenic tissues [38-42]. Poorly differentiated CRCs are more aggressive, does not have an efficient chemotherapy response [43], and shows high proliferation rates and metastasis, having an important impact in survival and prognosis of patients [44].

Our findings demonstrate that SOX9/BCL2L1 axis is present in undifferentiated CRC cell lines, and this could be related to a more aggressive phenotype that resists cell death.

SOX9 downregulation enhances ABT-737-induced apoptosis, but not autophagy in HCT-116.

In order to determine if downregulation of BCL2L1 given by SOX9 silencing produces changes in cell survival, we used ABT-737 as a proapoptotic stimulus. ABT-737 mimics the action of the $\mathrm{BH}$-only protein $\mathrm{BAD}$ by binding the antiapoptotic proteins BCL-2, BCL-xL, and BCL-w and inhibits their function, promoting apoptosis [45]. We found that, in untreated cells, SOX9 silencing has no effect in apoptosis (Figure 4(a)). In cancer conditions, SOX9 and BCL2L1 are overexpressed when compared to normal colon cells (data not shown), and there is a direct relation in their expression since BCL2L1 is a target of SOX9 [46]. However, SOX9 silencing inhibits BCL2L1 expression partially, which can be not enough to induce apoptosis. Besides, there are multiple prosurvival mechanisms [47-50] working at the same time in cancer cells, providing signals that help cells to resist apoptosis. On the contrary, when we compared late apoptosis in ABT-737 treated cells, HCT-116 cells showed $39.2 \%$ of apoptosis $(p=0.0034)$, and this behavior is enhanced by silencing of SOX9 $(60.3 \%, p=0.0003)$, suggesting a summative inhibition of BCL2L1 expression. These finding suggest that SOX9 plays an important role in cell survival, increasing the expression of prosurvival molecules, such as BCL2L1 in CRC.

Given that BCL2L1 is also an important regulator in autophagy-related mechanisms that promotes cell survival, we evaluated LC3-II protein, a marker of autophagy. In this regard, it has been demonstrated that disruption of BCL2L1BECN1 complex increases the expression of LC3-II, giving as a result the formation of autophagosomes [51-53].

Our results showed that SOX9 silencing does not induce autophagosome formation on HCT-116 cells (Figure 5). Nonetheless, there are multiple mechanisms that are involved in autophagosome formation, involving several molecules such as Ulk1 and 2, Becn1, Atg, LC3, and Tfeb [54-59].

\section{Conclusions}

Differential expression analysis of SOX9-silenced HCT-116 cells showed seven main clusters: nucleosome core, transcription regulation, apoptosis regulation, beta-catenin-TFC complex assembly, cell cycle, zing finger, and DNA repair. APC and MYC showed the highest dysregulation, as well as histones and transcriptional regulators.

SOX9 silencing induced changes in expression patterns of prosurvival BCL2L1 and antisurvival CASP3, suggesting a SOX9 participation in cell survival. Interestingly, a consistent pattern in poorly and moderated-differentiated cell was found.

Finally, inhibition of BCL2L1 is enough to induce apoptosis; however, SOX9 silencing potentiates this effect, but not autophagy, indicating that cell survival could be modulated via SOX9/BCL2L1 axis. To the best of our knowledge, this is the first report suggesting that cell survival in poorly and moderated-differentiated CRC cell lines.

\section{Data Availability}

All data included in this work are available within the manuscript and supplementary materials.

\section{Conflicts of Interest}

The authors declare no conflicts of interest.

\section{Acknowledgments}

The authors acknowledge Consejo Nacional de Ciencia y Tecnología CONACYT for Mariana Avendaño-Félix (575985) and Erik Lizárraga-Verdugo (304939) fellowships. The authors acknowledge Consejo Nacional de Ciencia y Tecnología CONACYT (grant 290311) for funding.

\section{Supplementary Materials}

Figure S1: pathway analysis. Pathway with overrepresentation is the CRC pathway in which APC appears. Interestingly, APC expression is restored when SOX9 is silenced according to DE analysis. Figure S2: pathway analysis. Pathway with overrepresentation is the WNT signaling pathway in which APC appears. Interestingly, APC expression is restored when SOX9 is silenced according to DE analysis. Figure S3: string interactome (https://string-db. org/cgi/network.pl?taskId=Y8RKNUbzndpT). In Table 1, genes grouped in apoptosis cluster shows a network associating SOX9 with BCL2L1 through JUN. Besides, SOX9 is linked directly to CASP according to text mining and association of BCL2L1 and CASP3 is established based on experimental results. Table S1: differentially expressed coding genes between HCT-116 vs. HCT-116siSOX9 selected based on a fold-change of 2 in absolute value, the genes with an adjusted $p$ value $<0.01$. (Supplementary Materials)

\section{References}

[1] S. Ogino and M. Stampfer, "Lifestyle factors and microsatellite instability in colorectal cancer: the evolving field of molecular 
pathological epidemiology," JNCI Journal of the National Cancer Institute, vol. 102, no. 6, pp. 365-367, 2010.

[2] K. A. Cronin, A. J. Lake, S. Scott et al., "Annual report to the nation on the status of cancer, part I: national cancer statistics," Cancer, vol. 124, no. 13, pp. 2785-2800, 2018.

[3] B. Vogelstein, E. R. Fearon, S. R. Hamilton et al., "Genetic alterations during colorectal-tumor development," New England Journal of Medicine, vol. 319, no. 9, pp. 525-532, 1988.

[4] E. Marshman, P. D. Ottewell, C. S. Potten, and A. J. M. Watson, "Caspase activation during spontaneous and radiation-induced apoptosis in the murine intestine," The Journal of Pathology, vol. 195, no. 3, pp. 285-292, 2001.

[5] Z. Su, Z. Yang, Y. Xu, Y. Chen, and Q. Yu, "Apoptosis, autophagy, necroptosis, and cancer metastasis," Molecular Cancer, vol. 14, no. 1, 48 pages, 2015.

[6] D. Hanahan and R. A. Weinberg, "Hallmarks of cancer: the next generation," Cell, vol. 144, no. 5, pp. 646-674, 2011.

[7] A. Nagasaka, K. Kawane, H. Yoshida, and S. Nagata, "Apaf-1independent programmed cell death in mouse development," Cell Death \& Differentiation, vol. 17, no. 6, pp. 931-941, 2010.

[8] M. E. Guicciardi and G. J. Gores, "Life and death by death receptors," The FASEB Journal, vol. 23, no. 6, pp. 1625-1637, 2009.

[9] D. R. Green and G. Kroemer, "The pathophysiology of mitochondrial cell death," Science, vol. 305, no. 5684, pp. 626629, 2004.

[10] A. Pryczynicz, M. Gryko, K. Niewiarowska et al., "Bax protein may influence the invasion of colorectal cancer," World Journal of Gastroenterology, vol. 20, no. 5, pp. 1305-1310, 2014.

[11] L. H. Boise, M. González-García, C. E. Postema et al., "Bcl-x, a bcl-2-related gene that functions as a dominant regulator of apoptotic cell death," Cell, vol. 74, no. 4, pp. 597-608, 1993.

[12] R. J. Youle and A. Strasser, "The BCL-2 protein family: opposing activities that mediate cell death," Nature Reviews Molecular Cell Biology, vol. 9, no. 1, pp. 47-59, 2008.

[13] H. Zhang, J. Xue, P. Hessler et al., "Genomic analysis and selective small molecule inhibition identifies BCL-X(L) as a critical survival factor in a subset of colorectal cancer," Molecular Cancer, vol. 14, no. 1, p. 126, 2015.

[14] L. Topol, W. Chen, H. Song, T. F. Day, and Y. Yang, "Sox9 inhibits wnt signaling by promoting $\beta$-catenin phosphorylation in the nucleus," Journal of Biological Chemistry, vol. 284, no. 5, pp. 3323-3333, 2009.

[15] A. Jo, S. Denduluri, B. Zhang et al., "The versatile functions of Sox9 in development, stem cells, and human diseases," Genes \& Diseases, vol. 1, no. 2, pp. 149-161, 2014.

[16] P. Bastide, C. Pannequin, J. Pannequin et al., "Sox9 regulates cell proliferation and is required for paneth cell differentiation in the intestinal epithelium," Journal of Cell Biology, vol. 178, no. 4, pp. 635-648, 2007.

[17] J. W. Foster, M. S. Dominguez-Steglich, S. Guioli et al., "Campomelic dysplasia and autosomal sex reversal caused by mutations in an SRY-related gene," Nature, vol. 372, no. 6506, pp. 525-530, 1994.

[18] H. Liu, Z. Liu, and B. Jiang, "SOX9 overexpression promotes glioma metastasis via wnt/b -catenin signaling," Cell Biochemistry and Biophysics, vol. 73, no. 1, pp. 205-212, 2015.

[19] G. Domenici, I. Aurrekoetxea-Rodríguez, B. M. Simões et al., "A Sox2-Sox9 signalling axis maintains human breast luminal progenitor and breast cancer stem cells," Oncogene, vol. 38, no. 17, pp. 3151-3169, 2019.

[20] J. Q. Huang, F. K. Wei, X. L. Xu et al., "SOX9 drives the epithelial-mesenchymal transition in non-small-cell lung cancer through the Wnt/beta-catenin pathway," Journal of Translational Medicine, vol. 17, no. 1, p. 143, 2019.

[21] D. Grimm, J. Bauer, P. Wise et al., "The role of SOX family members in solid tumours and metastasis," Seminars in Cancer Biology, 2019, In press.

[22] R. A. Irizarry, B. Hobbs, F. Collin et al., "Exploration, normalization, and summaries of high density oligonucleotide array probe level data," Biostatistics, vol. 4, no. 2, pp. 249-264, 2003.

[23] Y. Benjamini and Y. Hochberg, "Controlling the false discovery rate: a practical and powerful approach to multiple testing," Journal of the Royal Statistical Society: Series B (Methodological), vol. 57, no. 1, pp. 289-300, 1995.

[24] D. W. Huang, B. T. Sherman, and R. A. Lempicki, "Systematic and integrative analysis of large gene lists using DAVID bioinformatics resources," Nature Protocols, vol. 4, no. 1, pp. 44-57, 2009.

[25] D. W. Huang, B. T. Sherman, X. Zheng et al., "Extracting biological meaning from large gene lists with DAVID," Current Protocols in Bioinformatics, vol. 27, no. 1, pp. 1-13, 2009.

[26] Z. Tang, C. Li, B. Kang, G. Gao, C. Li, and Z. Zhang, "GEPIA: a web server for cancer and normal gene expression profiling and interactive analyses," Nucleic Acids Research, vol. 45, no. W1, pp. W98-W102, 2017.

[27] Y. P. Wan, M. Xi, H. C. He et al., "Expression and clinical significance of SOX9 in renal cell carcinoma, bladder cancer and penile cancer," Oncology Research and Treatment, vol. 40, no. 1-2, pp. 15-20, 2017.

[28] B. Lü, Y. Fang, J. Xu et al., "Analysis of SOX9 expression in colorectal cancer," American Journal of Clinical Pathology, vol. 130, no. 6, pp. 897-904, 2008.

[29] L. Montorsi, F. Guizzetti, C. Alecci et al., "Loss of ZFP36 expression in colorectal cancer correlates to $\mathrm{wnt} / \beta$-catenin activity and enhances epithelial-to-mesenchymal transition through upregulation of ZEB1, SOX9 and MACC1," Oncotarget, vol. 7, no. 37, pp. 59144-59157, 2016.

[30] E. Carrasco-Garcia, L. Lopez, P. Aldaz et al., "SOX9-regulated cell plasticity in colorectal metastasis is attenuated by rapamycin," Scientific Reports, vol. 6, Article ID 32350, 2016.

[31] R. Fodde, "The APC gene in colorectal cancer," European Journal of Cancer, vol. 38, no. 7, pp. 867-871, 2002.

[32] J. M. Adams and S. Cory, "The bcl-2 protein family: arbiters of cell survival," Science, vol. 281, no. 5381, pp. 1322-1326, 1998.

[33] R. Yagi, D. McBurney, and W. E. Horton, "Bcl-2 positively regulates Sox9-dependent chondrocyte gene expression by suppressing the MEK-ERK1/2 signaling pathway," Journal of Biological Chemistry, vol. 280, no. 34, pp. 30517-30525, 2005.

[34] H. Chen, C. C. Garbutt, D. Spentzos, E. Choy, F. J. Hornicek, and Z. Duan, "Expression and therapeutic potential of SOX9 in chordoma," Clinical Cancer Research, vol. 23, no. 17, pp. 5176-5186, 2017.

[35] X. Yuan, J. Li, C. Coulouarn et al., "SOX9 expression decreases survival of patients with intrahepatic cholangiocarcinoma by conferring chemoresistance," British Journal of Cancer, vol. 119, no. 11, pp. 1358-1366, 2018.

[36] J.-C. Larsimont, K. K. Youssef, A. Sánchez-Danés et al., “Sox9 controls self-renewal of oncogene targeted cells and links tumor initiation and invasion," Cell Stem Cell, vol. 17, no. 1, pp. 60-73, 2015.

[37] J. C. Francis, A. Capper, J. Ning, E. Knight, J. D. Bono, and A. Swain, "SOX9 is a driver of aggressive prostate cancer by promoting invasion, cell fate and cytoskeleton alterations and epithelial to mesenchymal transition," vol. 9, no. 7, pp. 7604-7615, 2018. 
[38] M. Vogler, "Targeting BCL2-proteins for the treatment of solid tumours," Advances in Medicine, vol. 2014, Article ID 943648, 14 pages, 2014.

[39] X. Yu, L. Yang, M. J. Cairns et al., "Chemosensitization of solid tumors by inhibition of Bcl-xL expression using DNAzyme," Oncotarget, vol. 5, no. 19, pp. 9039-9048, 2014.

[40] Y. Sun, X. Y. Chen, J. Liu et al., "Differential caspase-3 expression in noncancerous, premalignant and cancer tissues of stomach and its clinical implication," Cancer Detection and Precention, vol. 30, no. 2, pp. 168-173, 2006.

[41] X. Pu, S. J. Storr, Y. Zhang et al., "Caspase- 3 and caspase- 8 expression in breast cancer: caspase- 3 is associated with survival," Apoptosis, vol. 22, no. 3, pp. 357-368, 2017.

[42] P.-F. Liu, Y.-C. Hu, B.-H. Kang et al., "Expression levels of cleaved caspase-3 and caspase-3 in tumorigenesis and prognosis of oral tongue squamous cell carcinoma," PLoS One, vol. 12, no. 7, Article ID e0180620, 2017.

[43] L. Shen, X. Qu, H. Li et al., "NDRG2 facilitates colorectal cancer differentiation through the regulation of Skp2-p21/p27 axis," Oncogene, vol. 37, no. 13, pp. 1759-1774, 2018.

[44] K. Derwinger, K. Kodeda, E. Bexe-Lindskog, and H. Taflin, "Tumour differentiation grade is associated with TNM staging and the risk of node metastasis in colorectal cancer," Acta Oncologica, vol. 49, no. 1, pp. 57-62, 2010.

[45] T. Oltersdorf, S. W. Elmore, A. R. Shoemaker et al., "An inhibitor of Bcl-2 family proteins induces regression of solid tumours," Nature, vol. 435, no. 7042, pp. 677-681, 2005.

[46] Genecards, BCL2L1 gene, 2020, https://www.genecards.org/ cgi-bin/carddisp.pl?gene=BCL2L1.

[47] L. K. Boroughs and R. J. DeBerardinis, "Metabolic pathways promoting cancer cell survival and growth," Nature Cell Biology, vol. 17, no. 4, pp. 351-359, 2015.

[48] P. Paoli, E. Giannoni, and P. Chiarugi, "Anoikis molecular pathways and its role in cancer progression," Biochimica et Biophysica Acta (BBA)-Molecular Cell Research, vol. 1833, no. 12 , pp. 3481-3498, 2013.

[49] G. Song, G. Ouyang, and S. Bao, "The activation of Akt/PKB signaling pathway and cell survival," Journal of Cellular and Molecular Medicine, vol. 9, no. 1, pp. 59-71, 2005.

[50] C. W. Yun and S. H. Lee, "The roles of autophagy in cancer," International Journal of Molecular Sciences, vol. 19, no. 11, 3466 pages, 2018.

[51] M. C. Maiuri, G. Le Toumelin, A. Criollo et al., "Functional and physical interaction between Bcl-XL and a BH3-like domain in Beclin-1," The EMBO Journal, vol. 26, no. 10, pp. 2527-2539, 2007.

[52] N. Mizushima, A. Yamamoto, M. Matsui, T. Yoshimori, and Y. Ohsumi, "In vivo analysis of autophagy in response to nutrient starvation using transgenic mice expressing a fluorescent autophagosome marker," Molecular Biology of the Cell, vol. 15, no. 3, pp. 1101-1111, 2004.

[53] Y. Mei, K. Glover, M. Su, and S. C. Sinha, "Conformational flexibility of BECN1: essential to its key role in autophagy and beyond," Protein Science, vol. 25, no. 10, pp. 1767-1785, 2016.

[54] L. Liu, Z. Tao, L. D. Zheng et al., "FoxO1 interacts with transcription factor EB and differentially regulates mitochondrial uncoupling proteins via autophagy in adipocytes," Cell Death Discovery, vol. 2, no. 1, 16066 pages, 2016.

[55] J. Fullgrabe, D. J. Klionsky, and B. Joseph, "The return of the nucleus: transcriptional and epigenetic control of autophagy," Nature Reviews Molecular Cell Biology, vol. 15, no. 1, pp. 65-74, 2014.

[56] N. Hariharan, Y. Maejima, J. Nakae, J. Paik, R. A. Depinho, and J. Sadoshima, "Deacetylation of FoxO by Sirt1 plays an essential role in mediating starvation-induced autophagy in cardiac myocytes," Circulation Research, vol. 107, no. 12, pp. 1470-1482, 2010.

[57] S. Sinha and B. Levine, "The autophagy effector Beclin 1: a novel BH3-only protein," Oncogene, vol. 27, no. S1, pp. S137-S148, 2008.

[58] Y. Chen, L. Lv, H. Pi et al., "Dihydromyricetin protects against liver ischemia/reperfusion induced apoptosis via activation of FOXO3a-mediated autophagy," Oncotarget, vol. 7, no. 47, pp. 76508-76522, 2016.

[59] B. T. O’Neill, G. Bhardwaj, C. M. Penniman et al., "FoxO transcription factors are critical regulators of diabetes-related muscle atrophy," Diabetes, vol. 68, no. 3, pp. 556-570, 2019. 